\title{
Tidal perturbations of linear, isentropic oscillations in components of circular-orbit close binaries
}

\section{Validity of the perturbation method applied to equilibrium tides}

\author{
K. Reyniers and P. Smeyers
}

Instituut voor Sterrenkunde, Katholieke Universiteit Leuven, Belgium

Received 7 May 2003 / Accepted 7 July 2003

\begin{abstract}
The paper is devoted to a verification of the validity of a first-order perturbation method that was developed in an earlier paper and allows one to determine the effects of an equilibrium tide on linear, isentropic oscillations of a component in a close binary. The verification is done by a comparison between results obtained by the perturbation method and results obtained in other ways, in the cases of two simple models: the compressible equilibrium sphere with uniform mass density and the polytropic model with index $n=3$. In the first case, a comparison is made with second-harmonic oscillations in compressible Jeans spheroids with a small eccentricity, and in the second case, with results determined by Saio (1981) for a rotationally and tidally distorted polytrope. For the comparison, the second-harmonic oscillations of the incompressible and the compressible Jeans spheroids are redetermined by means of a method of direct integration of the governing equations which has the advantage of yielding exact analytical solutions of the eigenfrequency equations.
\end{abstract}

Key words. stars: binaries: close - stars: oscillations - methods: analytical

\section{Introduction}

In a preceding paper (Reyniers \& Smeyers 2003), hereafter referred to as Paper I, we have developed a perturbation method in order to determine the effects of an equilibrium tide exerted by a companion on linear, isentropic oscillations of a uniformly rotating star that is a component of a close binary. The perturbation method applies to any physical model of a non-rotating star in hydrostatic equilibrium and takes the star's tidal distortion into account in a first-order approximation. A limitation of the perturbation method is that the effects of the Coriolis force and the centrifugal force are left out of consideration.

Here our aim is to test the validity of the perturbation method by comparing results derived by means of the perturbation method with results derived by other means, in the cases of two simple models: the compressible equilibrium sphere with uniform mass density, shortly called the compressible homogeneous model, and the polytropic model with index $n=3$. In the first case, we compare with second-harmonic oscillations established for compressible Jeans spheroids with a small eccentricity, and in the second case, with results determined by Saio (1981) for a rotationally and tidally distorted polytrope by means of a perturbation method that has been developed earlier by Smeyers \& Denis (1971) and Denis (1972).

Send offprint requests to: $\mathrm{P}$. Smeyers, e-mail: Paul.Smeyers@ster.kuleuven.ac.be
The Jeans spheroids are static equilibrium configurations with uniform mass density that are subject to the equilibrium tide generated by another body, which is considered to be a point mass. For our purpose, we redetermine the secondharmonic oscillations successively of the incompressible and the compressible Jeans spheroids by a method of direct integration of the governing equations similar to the method that has been developed by Smeyers (1986) for the determination of oscillation modes of the incompressible MacLaurin spheroids. Compared to the virial method of Chandrasekhar \& Lebovitz (1963), our method has the advantage of yielding exact analytical solutions of the eigenfrequency equations, which are of particular interest here in the limiting case of weak equilibrium tides. The redetermination of the second-harmonic oscillations of the Jeans spheroids is done in Sect. 2.

Section 3 is devoted to the comparison of results derived by means of the perturbation method in the cases of the compressible homogeneous model and the polytropic model with index $n=3$. Within the framework of this comparison, we regard the Jeans spheroids as tidally distorted equilibrium configurations that rotate uniformly in synchronization with an orbiting body without being affected by the rotation.

In Sect. 4, concluding remarks are presented.

We adopt the same notations as in Paper I. In particular, we use two different systems of spherical coordinates with origin at the star's mass centre: a system of spherical coordinates $r$, $\theta, \phi$ whose polar axis coincides with the star's rotation axis, 


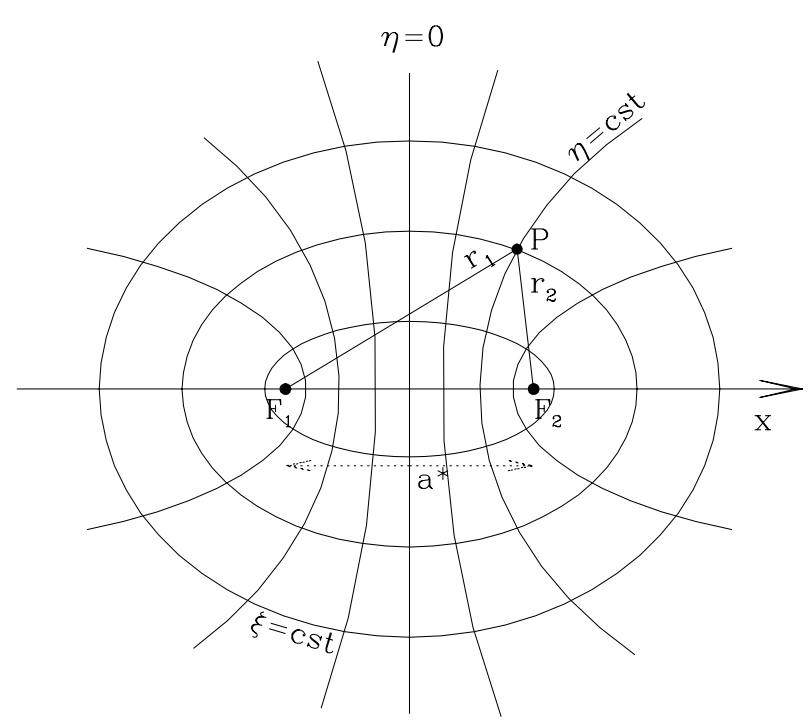

Fig. 1. An orthogonal system of confocal ellipses and hyperbolas, which generates coordinate surfaces of constant $\xi$ and coordinate surfaces of constant $\eta$ by rotation around the axis joining the common foci.

and a system of spherical coordinates $r, \theta^{*}, \phi^{*}$ whose polar axis coincides with the axis joining the star's mass centre and the companion. With the first system of spherical coordinates, spherical harmonics $Y_{\ell}^{m}(\theta, \phi)$ of degree $\ell$ are associated, and with the second system, spherical harmonics $Y_{\ell}^{\widetilde{m}}\left(\theta^{*}, \phi^{*}\right)$ of the same degree.

\section{The Jeans spheroids and their second-harmonic oscillations}

\subsection{Equilibrium structure of the Jeans spheroids}

Let $O x y z$ be an inertial frame of reference whose origin coincides with the mass centre of the Jeans spheroid and whose $x$-axis points towards the other body. With respect to this frame of reference, we introduce a system of prolate spheroidal coordinates $\xi, \eta, \phi^{*}$ whose axis of revolution coincides with the $x$ axis. A system of prolate spheroidal coordinates is defined by an orthogonal system of confocal ellipses and hyperbolas that is rotating about the axis joining the common foci, as is illustrated by Fig. 1. The transformation formulae from the Cartesian coordinates $x, y, z$ to the prolate spheroidal coordinates $\xi, \eta, \phi^{*}$ are

$x=\frac{1}{2} a^{*}\left[\xi \eta \cos \left[\Omega\left(t-t_{0}\right)\right]\right.$

$$
\left.-\sqrt{\left(\xi^{2}-1\right)\left(1-\eta^{2}\right)} \cos \phi^{*} \sin \left[\Omega\left(t-t_{0}\right)\right]\right],
$$

$y=\frac{1}{2} a^{*}\left[\xi \eta \sin \left[\Omega\left(t-t_{0}\right)\right]\right.$

$$
\begin{aligned}
& \left.\quad+\sqrt{\left(\xi^{2}-1\right)\left(1-\eta^{2}\right)} \cos \phi^{*} \cos \left[\Omega\left(t-t_{0}\right)\right]\right] \\
& z=\frac{1}{2} a^{*} \sqrt{\left(\xi^{2}-1\right)\left(1-\eta^{2}\right)} \sin \phi^{*} .
\end{aligned}
$$

Here, $a^{*} / 2$ is the distance, from the centre, of the common foci of the spheroidal and hyperbolic coordinate surfaces.

For the construction of the Jeans spheroids, we apply a procedure similar to the one adopted by Smeyers (1986) for the construction of uniformly rotating MacLaurin spheroids. We suppose that the surface of the Jeans spheroid coincides with the spheroidal coordinate surface $\xi=\xi_{\mathrm{S}}$.

The internal gravitational potential is derived from Poisson's integral formula

$\Phi(\boldsymbol{r})=-G \int_{V} \frac{\rho\left(\boldsymbol{r}^{\prime}\right)}{\left|\boldsymbol{r}^{\prime}-\boldsymbol{r}\right|} \mathrm{d} V\left(\boldsymbol{r}^{\prime}\right)$

by the use of the expansion for the reciprocal of the distance between a point with position vector $\boldsymbol{r}^{\prime}$ and spheroidal coordinates $\xi^{\prime}, \eta^{\prime}, \phi^{* \prime}$ and the point with position vector $\boldsymbol{r}$ and spheroidal coordinates $\xi, \eta, \phi^{*}$

$$
\begin{aligned}
\left|\boldsymbol{r}^{\prime}-\boldsymbol{r}\right|^{-1}= & \frac{2}{a^{*}} \sum_{n=0}^{\infty}(2 n+1) \sum_{m=0}^{n} \epsilon_{m} i^{m}\left[\frac{(n-m) !}{(n+m) !}\right]^{2} \\
& \times \cos \left[m\left(\phi^{* \prime}-\phi^{*}\right)\right] f_{n, m}\left(\xi, \xi^{\prime}\right) P_{n}^{m}(\eta) P_{n}^{m}\left(\eta^{\prime}\right),
\end{aligned}
$$

where

$$
\left.\begin{array}{l}
f_{n, m}\left(\xi, \xi^{\prime}\right)=P_{n}^{m}\left(\xi^{\prime}\right) Q_{n}^{m}(\xi) \text { as } \xi^{\prime}<\xi, \\
f_{n, m}\left(\xi, \xi^{\prime}\right)=P_{n}^{m}(\xi) Q_{n}^{m}\left(\xi^{\prime}\right) \text { as } \xi^{\prime}>\xi,
\end{array}\right\}
$$

and $\epsilon_{m}$ is Neuman's factor defined as

$\epsilon_{0}=1, \epsilon_{m}=2$ for $m=1,2,3, \ldots$

The $Q_{n}^{m}(\xi)$ are Legendre functions of the second kind (Morse $\&$ Feshbach 1953, Sect. 10.3).

After integration over the azimuthal angle $\phi^{* \prime}$, the expansion for the internal gravitational potential reduces to

$$
\begin{aligned}
& \Phi(\xi, \eta)=-\frac{\pi}{2} G \rho a^{* 2} \sum_{n=0}^{\infty}(2 n+1) \\
& \quad \times\left[P_{n}(\eta) Q_{n}(\xi) \int_{1}^{\xi} \mathrm{d} \xi^{\prime} \int_{-1}^{1} \mathrm{~d} \eta^{\prime}\left(\xi^{\prime 2}-\eta^{\prime 2}\right) P_{n}\left(\eta^{\prime}\right) P_{n}\left(\xi^{\prime}\right)\right. \\
& \left.\quad+P_{n}(\eta) P_{n}(\xi) \int_{\xi}^{\xi \mathrm{s}} \mathrm{d} \xi^{\prime} \int_{-1}^{1} \mathrm{~d} \eta^{\prime}\left(\xi^{\prime 2}-\eta^{\prime 2}\right) P_{n}\left(\eta^{\prime}\right) Q_{n}\left(\xi^{\prime}\right)\right] .
\end{aligned}
$$

Next, after integration over the coordinates $\xi^{\prime}$ and $\eta^{\prime}$ and summation over all values of $n$, one finds

$$
\begin{aligned}
\Phi(\xi, \eta)= & -\frac{\pi}{8} G \rho a^{* 2}\left\{A_{1}\left(\xi^{2}+\eta^{2}-3 \xi^{2} \eta^{2}+1\right)\right. \\
& \left.+4\left(\xi_{\mathrm{S}}^{2}-\xi^{2} \eta^{2}\right)\right\}
\end{aligned}
$$

with

$$
A_{1}=\xi_{\mathrm{S}}\left[\left(\xi_{\mathrm{S}}^{2}-1\right) \ln \frac{\xi_{\mathrm{S}}+1}{\xi_{\mathrm{S}}-1}-2 \xi_{\mathrm{S}}\right] .
$$

In terms of the prolate spheroidal coordinates $\xi$ and $\eta$, the potential generating a second-degree equilibrium tide takes the form

$\varepsilon_{\mathrm{T}} W(\xi, \eta)=\varepsilon_{\mathrm{T}} \frac{G M_{1}}{R_{1}^{3}} \frac{a^{* 2}}{8}\left(\xi^{2}+\eta^{2}-3 \xi^{2} \eta^{2}-1\right)$. 
We impose the condition that the equilibrium configuration be a prolate spheroid by requiring that

$\left[\frac{\partial}{\partial \eta}\left(\Phi+\varepsilon_{\mathrm{T}} W\right)\right]_{\xi_{\mathrm{s}}}=0$

In terms of the prolate spheroidal coordinates, the condition takes the form

$\pi G \rho\left[A_{1}\left(1-3 \xi_{\mathrm{S}}^{2}\right)-4 \xi_{\mathrm{S}}^{2}\right]=\varepsilon_{\mathrm{T}} \frac{G M_{1}}{R_{1}^{3}}\left(1-3 \xi_{\mathrm{S}}^{2}\right)$.

The value of $\xi_{\mathrm{S}}$ being related to the eccentricity $e$ of the surface of the Jeans spheroid as

$\xi_{\mathrm{S}}=\frac{1}{e}$,

the latter condition links the eccentricity of the spheroidal surface of the equilibrium configuration to the strength of the tidal action of the disturbing body. By setting

$\mu=\frac{G M_{2}}{a^{3}}$

and using the definition

$\varepsilon_{\mathrm{T}}=\left(\frac{R_{1}}{a}\right)^{3} \frac{M_{2}}{M_{1}}$,

where $a$ is the radius of the other body's relative orbit, one derives the relation

$\mu(e)=\pi G \rho \frac{1-e^{2}}{e^{2}}\left(\frac{1}{e} \ln \frac{1+e}{1-e}-\frac{6}{3-e^{2}}\right)$,

which corresponds to a relation given by Chandrasekhar (1969, Chap. 8). The variation of $\mu$ as a function of the eccentricity $e$ of the surface of the spheroid is represented in Fig. 2. The function $\mu(e)$ attains its maximum value $\mu=0.12554$ at the eccentricity of the surface $e=0.88303$. For small values of the eccentricity, the Taylor series near $e=0$ can be used

$\mu(e)=\frac{8}{45} \pi G \rho e^{2}\left[1+\frac{4}{21} e^{2}+O\left(e^{4}\right)\right]$.

The integration of the conditions of hydrostatic equilibrium

$\left.\begin{array}{l}\frac{1}{\rho} \frac{\partial P}{\partial \xi}=-\frac{\partial \Phi}{\partial \xi}-\varepsilon_{\mathrm{T}} \frac{\partial W}{\partial \xi}, \\ \frac{1}{\rho} \frac{\partial P}{\partial \eta}=-\frac{\partial \Phi}{\partial \eta}-\varepsilon_{\mathrm{T}} \frac{\partial W}{\partial \eta} .\end{array}\right\}$

Yields the pressure distribution

$$
\begin{aligned}
P= & \rho \frac{a^{* 2}}{8}\left\{\pi G \rho\left[A_{1}\left(\xi^{2}+\eta^{2}-3 \xi^{2} \eta^{2}\right)-4 \xi^{2} \eta^{2}\right]\right. \\
& \left.-\varepsilon_{\mathrm{T}} \frac{G M_{1}}{R_{1}^{3}}\left(\xi^{2}+\eta^{2}-3 \xi^{2} \eta^{2}\right)\right\}+C,
\end{aligned}
$$

where the constant $C$ is determined by the condition that the pressure vanishes at the equilibrium surface. The pressure distribution can then be simplified to

$$
P=\pi G \rho^{2} \frac{a^{* 2}}{2} \frac{\xi_{\mathrm{S}}^{2}-\eta^{2}}{3 \xi_{\mathrm{S}}^{2}-1}\left(\xi_{\mathrm{S}}^{2}-\xi^{2}\right) .
$$

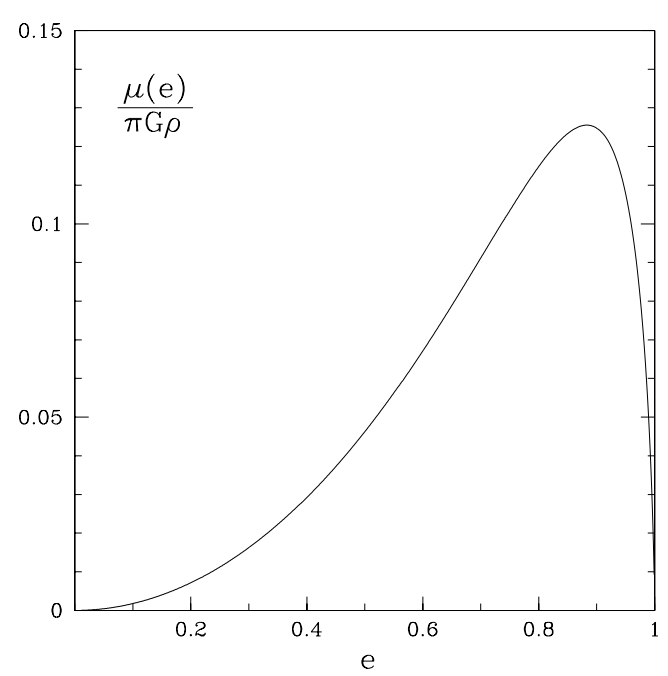

Fig. 2. The variation of $\mu$ as a function of the eccentricity $e$ of the surface of the Jeans spheroid.

\subsection{The second-harmonic oscillations of the incompressible Jeans spheroids}

Adapting the procedure of Smeyers (1986), we start constructing solutions from the centre of the Jeans spheroid in terms of spherical coordinates and then express the solutions in terms of the prolate spheroidal coordinates $\xi$ and $\eta$ in order to impose the boundary conditions at the surface of the Jeans spheroid. We concentrate on the second-harmonic oscillations in view of the comparison of our results with those of Chandrasekhar \& Lebovitz (1963).

Because of the axial symmetry of the Jeans spheroids with regard to the $x$-axis, it is appropriate to use a system of spherical coordinates $r, \theta^{*}, \phi^{*}$ whose polar axis coincides with this axis. The transformation formulae from the Cartesian coordinates $x, y, z$ defined in the inertial frame of reference to the spherical coordinates $r, \theta^{*}, \phi^{*}$ are

$$
\left.\begin{array}{rl}
x= & r\left\{\cos \theta^{*} \cos \left[\Omega\left(t-t_{0}\right)\right]\right. \\
& \left.-\sin \theta^{*} \cos \phi^{*} \sin \left[\Omega\left(t-t_{0}\right)\right]\right\} \\
y= & r\left\{\cos \theta^{*} \sin \left[\Omega\left(t-t_{0}\right)\right]\right. \\
& \left.+\sin \theta^{*} \cos \phi^{*} \cos \left[\Omega\left(t-t_{0}\right)\right]\right\} \\
z= & r \sin \theta^{*} \sin \phi^{*} .
\end{array}\right\}
$$

By means of the relations

$$
\left.\begin{array}{l}
r \cos \theta^{*}=\frac{a^{*}}{2} \xi \eta, \\
r \sin \theta^{*}=\frac{a^{*}}{2}\left[\left(\xi^{2}-1\right)\left(1-\eta^{2}\right)\right]^{1 / 2}, \\
r=\frac{a^{*}}{2}\left(\xi^{2}+\eta^{2}-1\right)^{1 / 2},
\end{array}\right\}
$$

the pressure distribution is expressed in terms of the spherical coordinates $r$ and $\theta^{*}$ as

$P=\frac{2 \pi G \rho^{2}}{3 \xi_{\mathrm{S}}^{2}-1}\left[r^{2}\left(\cos ^{2} \theta^{*}-\xi_{\mathrm{S}}^{2}\right)+\frac{a^{* 2}}{4} \xi_{\mathrm{S}}^{2}\left(\xi_{\mathrm{S}}^{2}-1\right)\right]$. 
The linear, isentropic oscillations of a Jeans spheroid that depend on time by a factor $\exp (\mathrm{i} \sigma t)$ are governed by the following equations:

the radial component of the vorticity equation

$\sigma^{2}\left[\frac{\partial}{\partial \theta^{*}}\left(\sin \theta^{*} \xi_{\phi^{*}}\right)-\frac{\partial \xi_{\theta^{*}}}{\partial \phi^{*}}\right]=\frac{1}{r} \frac{\partial}{\partial \phi^{*}}\left(\frac{\rho^{\prime}}{\rho^{2}} \frac{\partial P}{\partial \theta^{*}}\right)$,

the radial component of the equation of motion

$\sigma^{2} \xi_{r}=\frac{\partial \Phi^{\prime}}{\partial r}-\frac{\rho^{\prime}}{\rho^{2}} \frac{\partial P}{\partial r}+\frac{1}{\rho} \frac{\partial P^{\prime}}{\partial r}$

the equation for the divergence of the horizontal part of the Lagrangian displacement

$$
\begin{gathered}
\sigma^{2}\left[\frac{1}{\sin \theta^{*}} \frac{\partial}{\partial \theta^{*}}\left(\sin \theta^{*} \xi_{\theta^{*}}\right)+\frac{1}{\sin \theta^{*}} \frac{\partial \xi_{\phi^{*}}}{\partial \phi^{*}}\right]=-\frac{1}{r} \mathcal{L}^{2}\left(\Phi^{\prime}+\frac{P^{\prime}}{\rho}\right) \\
-\frac{1}{r \sin \theta^{*}} \frac{\partial}{\partial \theta^{*}}\left(\sin \theta^{*} \frac{\rho^{\prime}}{\rho^{2}} \frac{\partial P}{\partial \theta^{*}}\right)
\end{gathered}
$$

the equation expressing the mass conservation of the moving elements

$\frac{\rho^{\prime}}{\rho}+\alpha=0$

the energy equation expressing that the oscillations are isentropic

$\frac{\rho^{\prime}}{\rho}=\frac{1}{\Gamma_{1} P}\left(P^{\prime}+\frac{\partial P}{\partial r} \delta r+\frac{\partial P}{\partial \theta^{*}} \delta \theta^{*}\right)$,

the equation of Poisson

$\nabla^{2} \Phi^{\prime}=4 \pi G \rho^{\prime}$,

where $\xi_{r}, \xi_{\theta^{*}}, \xi_{\phi^{*}}$ are the components of the Lagrangian displacement with respect to the local orthonormal basis $\partial / \partial r$, $(1 / r)\left(\partial / \partial \theta^{*}\right), \quad\left[1 /\left(r \sin \theta^{*}\right)\right]\left(\partial / \partial \phi^{*}\right), \quad$ primed variables are Eulerian perturbations, and $\mathcal{L}^{2}$ is the Legendrian defined as

$\mathcal{L}^{2}=-\left[\frac{1}{\sin \theta^{*}} \frac{\partial}{\partial \theta^{*}}\left(\sin \theta^{*} \frac{\partial}{\partial \theta^{*}}\right)+\frac{1}{\sin ^{2} \theta^{*}} \frac{\partial^{2}}{\partial \phi^{* 2}}\right]$.

For the incompressible Jeans spheroids, one has that

$\alpha=0, \quad \Gamma_{1} \rightarrow \infty$,

so that

$\rho^{\prime}=0$,

and the Lagrangian displacement field is purely poloidal and can be represented as

$\boldsymbol{\xi}=\nabla \times \nabla \times\left(S \mathbf{1}_{r}\right)$

where $S\left(r, \theta^{*}, \phi^{*}\right)$ is a scalar function.

By expanding the scalar functions $S, P^{\prime}, \Phi^{\prime}$ in terms of spherical harmonics as

$f\left(r, \theta^{*}, \phi^{*}\right)=\sum_{\lambda=0}^{\infty} \sum_{\widetilde{\mu}=-\lambda}^{\lambda} f_{\lambda, \widetilde{\mu}}(r) Y_{\lambda}^{\widetilde{\mu}}\left(\theta^{*}, \phi^{*}\right)$, and using known properties of the spherical harmonics, one derives an infinite number of systems of differential equations, each involving three radial functions which are associated with a single spherical harmonic $Y_{\ell}^{\widetilde{m}}\left(\theta^{*}, \phi^{*}\right)$ :

$\frac{\mathrm{d} \chi \ell, \widetilde{m}}{\mathrm{~d} r}=\sigma^{2} \frac{\ell(\ell+1)}{r^{2}} S_{\ell, \widetilde{m}}$,

$\sigma^{2} \frac{\mathrm{d} S_{\ell, \widetilde{m}}}{\mathrm{~d} r}=\chi_{\ell, \widetilde{m}}$

$\frac{1}{r^{2}} \frac{\mathrm{d}}{\mathrm{d} r}\left(r^{2} \frac{\mathrm{d} \Phi_{\ell, \widetilde{m}}^{\prime}}{\mathrm{d} r}\right)-\frac{\ell(\ell+1)}{r^{2}} \Phi_{\ell, \widetilde{m}}^{\prime}=0$.

In these equations, we have set

$\chi_{\ell, \widetilde{m}}=\Phi_{\ell, \widetilde{m}}^{\prime}+\frac{P_{\ell, \widetilde{m}}^{\prime}}{\rho}$.

Because of the symmetry of the Jeans spheroids with regard to the axis pointing from their mass centre towards the other body, the azimuthal number $\widetilde{m}$ does not appear in the coefficients of the system of differential Eqs. (33).

For $\sigma^{2} \neq 0$, solutions of Eqs. (33) which satisfy the regularity conditions at $r=0$ are

$S_{\ell, \widetilde{m}}=A_{\ell, \widetilde{m}} r^{\ell+1}, \quad \chi_{\ell, \widetilde{m}}=B_{\ell, \widetilde{m}} r^{\ell}$,

$\Phi_{\ell, \widetilde{m}}^{\prime}=C_{\ell, \widetilde{m}} r^{\ell}$

Here, $A_{\ell, \widetilde{m}}, B_{\ell, \widetilde{m}}, C_{\ell, \widetilde{m}}$ are arbitrary constants, for which the relation holds

$B_{\ell, \widetilde{m}}=A_{\ell, \widetilde{m}} \sigma^{2}(\ell+1)$.

The infinite set of systems of differential Eqs. (33) can be satisfied, and the boundary conditions at the equilibrium surface of the Jeans spheroid can be fulfilled for a given azimuthal number $\widetilde{m}$, when one sets

$A_{2 \ell, \widetilde{m}}=0, \quad B_{2 \ell, \widetilde{m}}=0, \quad C_{2 \ell, \widetilde{m}}=0$,

for all $\ell$, except for $\ell=0$ and $\ell=2$.

The requirements that the pressure vanishes and the gravitational potential and its gradient are continuous at the perturbed surface of the Jeans spheroid lead to the conditions at the equilibrium surface $S$

$\left(P^{\prime}+\frac{\partial P}{\partial \xi} \delta \xi\right)_{S}=0$

$\left(\Phi^{\prime}\right)_{\mathrm{S}}=\left(\Phi_{\mathrm{e}}^{\prime}\right)_{\mathrm{S}}$

$\left(\frac{\partial \Phi^{\prime}}{\partial \xi}\right)_{\mathrm{S}}+\pi G \rho a^{* 2}\left(\frac{\xi^{2}-\eta^{2}}{\xi^{2}-1} \delta \xi\right)_{\mathrm{S}}=\left(\frac{\partial \Phi_{\mathrm{e}}^{\prime}}{\partial \xi}\right)_{\mathrm{S}}$, $\left(\frac{\partial \Phi^{\prime}}{\partial \eta}\right)_{\mathrm{S}}=\left(\frac{\partial \Phi_{e}^{\prime}}{\partial \eta}\right)_{\mathrm{S}}$

where $\Phi_{\mathrm{e}}^{\prime}$ is the Eulerian perturbation of the external gravitational potential.

We distinguish between the cases $\widetilde{m}=0, \widetilde{m}= \pm 1, \widetilde{m}= \pm 2$. 


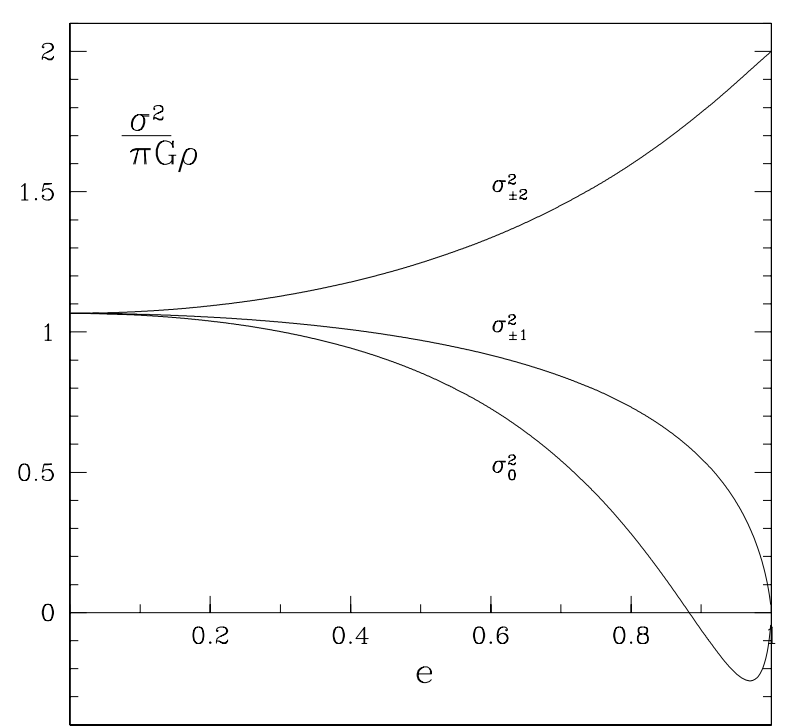

Fig. 3. The squares of the eigenfrequencies of the second-harmonic oscillations in the incompressible Jeans spheroids as functions of the eccentricity of the Jeans spheroid.

\subsubsection{The case $\widetilde{m}=0$}

The oscillations involve appropriate linear combinations of $\ell=$ 0 and $\ell=2$ terms. For $\widetilde{m}=0$ these are

$$
\left.\begin{array}{rl}
\delta r\left(r, \theta^{*}, \phi^{*}\right) & =6 A_{2,0} r P_{2}\left(\cos \theta^{*}\right), \\
\delta \theta^{*}\left(r, \theta^{*}, \phi^{*}\right) & =3 A_{2,0} \frac{\mathrm{d} P_{2}\left(\cos \theta^{*}\right)}{\mathrm{d} \theta^{*}}, \\
\delta \phi^{*}\left(r, \theta^{*}, \phi^{*}\right) & =0, \\
\Phi^{\prime}\left(r, \theta^{*}, \phi^{*}\right) & =C_{0,0}+C_{2,0} r^{2} P_{2}\left(\cos \theta^{*}\right), \\
P^{\prime}\left(r, \theta^{*}, \phi^{*}\right)= & \rho\left[\left(A_{0,0} \sigma^{2}-C_{0,0}\right)\right. \\
& \left.+\left(3 A_{2,0} \sigma^{2}-C_{2,0}\right) r^{2} P_{2}\left(\cos \theta^{*}\right)\right] .
\end{array}\right\}
$$

After transforming to prolate spheroidal coordinates and imposing the boundary conditions, one derives the equation for the square of the $(\widetilde{m}=0)$-eigenfrequency

$\sigma_{0}^{2}=12 \pi G \rho\left(1-e^{2}\right)\left[\frac{2}{\left(3-e^{2}\right)^{2}}-\frac{1}{e^{3}} Q_{2}\left(\frac{1}{e}\right)\right]$.

The variation of the eigenvalue $\sigma_{0}^{2}$ as a function of the eccentricity of the Jeans spheroid is represented in Fig. 3.

The eigenvalue $\sigma_{0}^{2}$ corresponds to the eigenvalue denoted as $\sigma_{3}^{2}$ by Chandrasekhar \& Lebovitz (1963) and Chandrasekhar (1969, Table 19). It is positive for $e<0.8830265$, vanishes at $e=0.8830265$, and is negative for $e>0.8830265$. Hence, the second-harmonic, axisymmetric mode is dynamically unstable for $e>0.8830265$. The critical value of the eccentricity corresponds to the value at which the parameter $\mu(e)$ determined by Eq. (14) attains its maximum value.

Near $e=0$, the following Taylor series applies:

$\sigma_{0}^{2}=\frac{16}{15} \pi G \rho\left[1-\frac{13}{21} e^{2}-\frac{13}{21} e^{4}+O\left(e^{6}\right)\right]$.

\subsubsection{The cases $\widetilde{m}= \pm 1$}

For $\widetilde{m}= \pm 1$, the solutions reduce to solutions associated with $\ell=2$ and take the form

$$
\begin{aligned}
\delta r\left(r, \theta^{*}, \phi^{*}\right)= & 6 A_{2, \pm 1} r P_{2}^{1}\left(\cos \theta^{*}\right) \exp \left( \pm \mathrm{i} \phi^{*}\right) \\
\delta \theta^{*}\left(r, \theta^{*}, \phi^{*}\right)= & 3 A_{2, \pm 1} \frac{\mathrm{d} P_{2}^{1}\left(\cos \theta^{*}\right)}{\mathrm{d} \theta^{*}} \exp \left( \pm \mathrm{i} \phi^{*}\right) \\
\delta \phi^{*}\left(r, \theta^{*}, \phi^{*}\right)= & \pm 3 \mathrm{i} A_{2, \pm 1} \frac{P_{2}^{1}\left(\cos \theta^{*}\right)}{\sin ^{2} \theta^{*}} \exp \left( \pm \mathrm{i} \phi^{*}\right) \\
\Phi^{\prime}\left(r, \theta^{*}, \phi^{*}\right)= & C_{2, \pm 1} r^{2} P_{2}^{1}\left(\cos \theta^{*}\right) \exp \left( \pm \mathrm{i} \phi^{*}\right) \\
P^{\prime}\left(r, \theta^{*}, \phi^{*}\right)= & \rho\left(3 A_{2, \pm 1} \sigma^{2}-C_{2, \pm 1}\right) \\
& \times r^{2} P_{2}^{1}\left(\cos \theta^{*}\right) \exp \left( \pm \mathrm{i} \phi^{*}\right)
\end{aligned}
$$

After transforming to prolate spheroidal coordinates and imposing the boundary conditions, one derives the equation for the square of the $(\widetilde{m}= \pm 1)$-eigenfrequency

$$
\sigma_{ \pm 1}^{2}=2 \pi G \rho\left(2-e^{2}\right)\left[\frac{2}{3-e^{2}}-\frac{\sqrt{1-e^{2}}}{e^{3}} Q_{2}^{1}\left(\frac{1}{e}\right)\right] \text {. }
$$

The eigenvalue $\sigma_{ \pm 1}^{2}$ is a double eigenvalue, since it depends only on the absolute value of the azimuthal number $\widetilde{m}$, i.e. $|\widetilde{m}|=1$. The variation of the eigenvalue $\sigma_{ \pm 1}^{2}$ as a function of the eccentricity of the Jeans spheroid is represented in Fig. 3.

The eigenvalue $\sigma_{ \pm 1}^{2}$ corresponds to the eigenvalue denoted as $\sigma_{1}^{2}$ by Chandrasekhar \& Lebovitz (1963) and Chandrasekhar (1969, Table 19) and is positive for all values of the eccentricity of the Jeans spheroid.

Near $e=0$, the following Taylor series applies:

$$
\sigma_{ \pm 1}^{2}=\frac{16}{15} \pi G \rho\left[1-\frac{13}{42} e^{2}-\frac{11}{63} e^{4}+O\left(e^{6}\right)\right] .
$$

\subsubsection{The cases $\widetilde{m}= \pm 2$}

For $\widetilde{m}= \pm 2$, the solutions also involve only $\ell=2$ terms, so that they take the form

$$
\begin{aligned}
\delta r\left(r, \theta^{*}, \phi^{*}\right)= & 6 A_{2, \pm 2} r P_{2}^{2}\left(\cos \theta^{*}\right) \exp \left( \pm 2 \mathrm{i} \phi^{*}\right) \\
\delta \theta^{*}\left(r, \theta^{*}, \phi^{*}\right)= & 3 A_{2, \pm 2} \frac{\mathrm{d} P_{2}^{2}\left(\cos \theta^{*}\right)}{\mathrm{d} \theta^{*}} \exp \left( \pm 2 \mathrm{i} \phi^{*}\right) \\
\delta \phi^{*}\left(r, \theta^{*}, \phi^{*}\right)= & \pm 6 \mathrm{i} A_{2, \pm 2} \frac{P_{2}^{2}\left(\cos \theta^{*}\right)}{\sin ^{2} \theta^{*}} \exp \left( \pm 2 \mathrm{i} \phi^{*}\right) \\
\Phi^{\prime}\left(r, \theta^{*}, \phi^{*}\right)= & C_{2, \pm 2} r^{2} P_{2}^{2}\left(\cos \theta^{*}\right) \exp \left( \pm 2 \mathrm{i} \phi^{*}\right) \\
P^{\prime}\left(r, \theta^{*}, \phi^{*}\right)= & \rho\left(3 A_{2, \pm 2} \sigma^{2}-C_{2, \pm 2}\right) \\
& \times r^{2} P_{2}^{2}\left(\cos \theta^{*}\right) \exp \left( \pm 2 \mathrm{i} \phi^{*}\right)
\end{aligned}
$$

After transforming to prolate spheroidal coordinates and imposing the boundary conditions, one derives the equation for the square of the $(\widetilde{m}= \pm 2)$-eigenfrequency

$\sigma_{ \pm 2}^{2}=\pi G \rho\left[\frac{8}{3-e^{2}}+\frac{e^{2}-1}{e^{3}} Q_{2}^{2}\left(\frac{1}{e}\right)\right]$. 
The eigenvalue $\sigma_{+2}^{2}$ is another double eigenvalue, since it depends only on the absolute value of the azimuthal number $\widetilde{m}$, i.e. $|\widetilde{m}|=2$. The variation of $\sigma_{ \pm 2}^{2}$ as a function of the eccentricity of the Jeans spheroid is represented in Fig. 3.

The eigenvalue $\sigma_{ \pm 2}^{2}$ corresponds to the eigenvalue denoted as $\sigma_{2}^{2}$ by Chandrasekhar \& Lebovitz (1963) and Chandrasekhar (1969, Table 19) and is positive for all values of the eccentricity of the Jeans spheroid.

Near $e=0$, the following Taylor series applies:

$\sigma_{ \pm 2}^{2}=\frac{16}{15} \pi G \rho\left[1+\frac{13}{21} e^{2}+\frac{13}{63} e^{4}+O\left(e^{6}\right)\right]$.

The squares of the eigenfrequencies of the second-harmonic oscillations, are determined by Eqs. (40), (43), and (46) for any value of the eccentricity of the Jeans spheroid. As $e \rightarrow 0$, the Taylor series for the squares of the eigenfrequencies for small values of $e$, which are given by Eqs. (41), (44), and (47), tend to the common value

$\sigma^{2}=\frac{16}{15} \pi G \rho$

This value corresponds to the degenerate value of the squares of the eigenfrequencies of the second-degree Kelvin modes in the incompressible homogeneous model. Hence, the secondharmonic oscillations of the incompressible Jeans spheroids are the continuations of the second-degree Kelvin modes of the incompressible homogeneous equilibrium sphere. They remain associated with a single spherical harmonic, if the polar axis of the system of spherical coordinates coincides with the axis pointing from the mass centre of the Jeans spheroid towards the other body.

\subsection{Second-harmonic oscillations of the compressible Jeans spheroids}

\subsubsection{The governing equations}

The linear, isentropic oscillations of the compressible Jeans spheroids that depend on time by a factor $\exp (\mathrm{i} \sigma t)$ are also governed by Eqs. (22)-(27). The Lagrangian displacement field is however generally not divergence-free and is described by the more general representation

$\boldsymbol{\xi}=\nabla \Psi+\nabla \times \nabla \times\left(S \mathbf{1}_{r}\right)+\nabla \times\left(T \mathbf{1}_{r}\right)$,

where $\Psi\left(r, \theta^{*}, \phi^{*}\right), S\left(r, \theta^{*}, \phi^{*}\right), T\left(r, \theta^{*}, \phi^{*}\right)$ are scalar functions.

By expanding the scalar functions $\Psi, S, T$, and the Eulerian perturbations $P^{\prime}, \rho^{\prime}, \Phi^{\prime}$ in terms of spherical harmonics in the form given in Equality (32), setting

$$
\begin{aligned}
& \xi_{\ell, \widetilde{m}}(r)=\frac{\mathrm{d} \Psi_{\ell, \widetilde{m}}(r)}{\mathrm{d} r}+\frac{\ell(\ell+1)}{r^{2}} S_{\ell, \widetilde{m}}(r), \\
& \eta_{\ell, \widetilde{m}}(r)=\Psi_{\ell, \widetilde{m}}(r)+\frac{\mathrm{d} S_{\ell, \widetilde{m}}(r)}{\mathrm{d} r},
\end{aligned}
$$

multiplying by the complex conjugate of a spherical harmonic $Y_{\ell}^{\widetilde{m}}\left(\theta^{*}, \phi^{*}\right)$, integrating over a solid angle of $4 \pi$, and using known properties of the spherical harmonics, one derives the system of equations

$$
\begin{aligned}
& \sigma^{2} \ell(\ell+1) T_{\ell, \widetilde{m}}=\mathrm{i} \widetilde{m} K r^{2}\left[K^{-}(\ell-1, \widetilde{m}) \alpha_{\ell-1, \widetilde{m}}\right. \\
& \left.+K^{+}(\ell+1, \widetilde{m}) \alpha_{\ell+1, \widetilde{m}}\right], \\
& \frac{\mathrm{d} \chi_{\ell, \widetilde{m}}}{\mathrm{~d} r}=\sigma^{2} \xi_{\ell, \widetilde{m}}+K r \xi_{\mathrm{S}}^{2} \alpha_{\ell, \widetilde{m}}-K r\left[L^{-}(\ell-2, \widetilde{m}) \alpha_{\ell-2, \widetilde{m}}\right. \\
& \left.+L(\ell, \widetilde{m}) \alpha_{\ell, \widetilde{m}}+L^{+}(\ell+2, \widetilde{m}) \alpha_{\ell+2, \widetilde{m}}\right], \\
& \ell(\ell+1)\left(\sigma^{2} \eta_{\ell, \widetilde{m}}-\chi_{\ell, \widetilde{m}}\right) \\
& =K r^{2}\left\{(\ell+1) L^{-}(\ell-2, \widetilde{m}) \alpha_{\ell-2, \widetilde{m}}\right. \\
& +\left[(\ell+3) L(\ell, \widetilde{m})-(\ell+|\widetilde{m}|) K^{-}(\ell-1, \widetilde{m})-1\right] \alpha_{\ell, \widetilde{m}} \\
& +\left[(\ell+5) L^{+}(\ell+2, \widetilde{m})\right. \\
& \left.\left.-(\ell+|\widetilde{m}|+2) K^{+}(\ell+1, \widetilde{m})\right] \alpha_{\ell+2, \widetilde{m}}\right\}, \\
& \frac{\mathrm{d}}{\mathrm{d} r}\left(r^{2} \xi_{\ell, \widetilde{m}}\right)=r^{2} \alpha_{\ell, \widetilde{m}}+\ell(\ell+1) \eta_{\ell, \widetilde{m}}, \\
& \chi_{\ell, \widetilde{m}}-\Phi_{\ell, \widetilde{m}}^{\prime}+K\left\{L(\ell, \widetilde{m}) r\left(\xi_{\ell, \widetilde{m}}-\ell \frac{\eta_{\ell, \widetilde{m}}}{r}+\frac{\Gamma_{1}}{2} r \alpha_{\ell, \widetilde{m}}\right)\right. \\
& +L^{-}(\ell-2, \widetilde{m}) r\left[\xi_{\ell-2, \widetilde{m}}-(\ell-2) \frac{\eta_{\ell-2, \widetilde{m}}}{r}+r \frac{\Gamma_{1}}{2} \alpha_{\ell-2, \widetilde{m}}\right] \\
& +L^{+}(\ell+2, \widetilde{m}) r\left[\xi_{\ell+2, \widetilde{m}}+(\ell+3) \frac{\eta_{\ell+2, \widetilde{m}}}{r}+r \frac{\Gamma_{1}}{2} \alpha_{\ell+2, \widetilde{m}}\right] \\
& +K^{-}(\ell-1, \widetilde{m})\left[(\ell+|\widetilde{m}|) \eta_{\ell, \widetilde{m}}-\mathrm{i} \widetilde{m} T_{\ell-1, \widetilde{m}}\right] \\
& -\mathrm{i} \widetilde{m} K^{+}(\ell+1, \widetilde{m}) T_{\ell+1, \widetilde{m}}-\xi_{\mathrm{S}}^{2} r \xi_{\ell, \widetilde{m}} \\
& \left.+\xi_{\mathrm{S}}^{2} \frac{\Gamma_{1}}{2}\left[-r^{2}+\frac{a^{* 2}}{4}\left(\xi_{\mathrm{S}}^{2}-1\right)\right] \alpha_{\ell, \widetilde{m}}\right\}=0, \\
& \frac{1}{r^{2}} \frac{\mathrm{d}}{\mathrm{d} r}\left(r^{2} \frac{\mathrm{d} \Phi_{\ell, \widetilde{m}}^{\prime}}{\mathrm{d} r}\right)-\frac{\ell(\ell+1)}{r^{2}} \Phi_{\ell, \widetilde{m}}^{\prime}=-4 \pi G \rho \alpha_{\ell, \widetilde{m}},
\end{aligned}
$$

where the following abbreviations are used:

$$
\begin{aligned}
& K=\frac{4 \pi G \rho}{3 \xi_{\mathrm{S}}^{2}-1} \\
& K^{-}(\ell, \widetilde{m})=\frac{\ell-|\widetilde{m}|+1}{2 \ell+1}, \quad K^{+}(\ell, \widetilde{m})=\frac{\ell+|\widetilde{m}|}{2 \ell+1} \\
& L^{-}(\ell, \widetilde{m})=K^{-}(\ell, \widetilde{m}) K^{-}(\ell+1, \widetilde{m}), \\
& L^{+}(\ell, \widetilde{m})=K^{+}(\ell-1, \widetilde{m}) K^{+}(\ell, \widetilde{m}) \\
& L(\ell, \widetilde{m})=K^{-}(\ell, \widetilde{m}) K^{+}(\ell+1, \widetilde{m}) \\
& \quad+K^{-}(\ell-1, \widetilde{m}) K^{+}(\ell, \widetilde{m}) .
\end{aligned}
$$

Replacing $\ell$ with $\ell-1$ in Eq. (51), we obtain

$$
\begin{aligned}
& \sigma^{2} \ell(\ell-1) T_{\ell-1, \widetilde{m}}=\mathrm{i} \widetilde{m} K r^{2}\left[K^{-}(\ell-2, \widetilde{m}) \alpha_{\ell-2, \widetilde{m}}\right. \\
& \left.\quad+K^{+}(\ell, \widetilde{m}) \alpha_{\ell, \widetilde{m}}\right], \quad|\widetilde{m}| \leq \ell+1 .
\end{aligned}
$$

Equations (52)-(56) and (58) form a system of three differential equations and three algebraic equations for the six unknown functions $\xi_{\ell, \widetilde{m}}(r), \eta_{\ell, \widetilde{m}}(r), \alpha_{\ell, \widetilde{m}}(r), \chi_{\ell, \widetilde{m}}(r), \Phi_{\ell, \widetilde{m}}^{\prime}(r), T_{\ell-1, \widetilde{m}}(r)$, which are associated with the degree $\ell$ and the azimuthal number $\widetilde{m}$. Each system of equations is coupled with the systems of equations associated with the same azimuthal number $\widetilde{m}$ but 
with the degrees $\ell-2$ and $\ell+2$, because of the presence of the unknown functions $\xi_{\ell \pm 2, \widetilde{m}}(r), \eta_{\ell \pm 2, \widetilde{m}}(r), \alpha_{\ell \pm 2, \widetilde{m}}(r), T_{\ell+1, \widetilde{m}}(r)$.

\subsubsection{Solutions}

One readily verifies that the non-axisymmetric secondharmonic solutions for the incompressible Jeans spheroids are also solutions for the compressible Jeans spheroids. Therefore, the search for second-harmonic solutions for the compressible Jeans spheroids can be restricted to a search for axisymmetric second-harmonic solutions. We proceed as follows.

The infinite number of systems of Eqs. (52)-(56) and (58) must be satisfied. To this end, we set

$\left.\begin{array}{l}\xi_{\ell, \widetilde{m}}=0, \quad \eta_{\ell, \widetilde{m}}=0, \quad \alpha_{\ell, \widetilde{m}}=0, \\ \chi_{\ell, \widetilde{m}}=0, \quad \Phi_{\ell, \widetilde{m}}^{\prime}=0, \quad T_{\ell+1, \widetilde{m}}=0\end{array}\right\}$

for all $\ell$, except for $\ell=0$ and $\ell=2$.

First, the system of equations associated with $\ell=4$ then reduces to

$T_{3,0}=0, \quad \alpha_{2,0}=0, \quad r \xi_{2,0}-2 \eta_{2,0}=0$.

Secondly, from the system of equations associated with $\ell=$ 2 , it follows that

$\left.\begin{array}{l}T_{1,0}=0, \quad \xi_{2,0}=A_{2,0}, \\ \eta_{2,0}=\frac{A_{2,0}}{2} r^{2}, \quad \alpha_{0,0}=D_{0,0}, \\ \Phi_{2,0}^{\prime}=C_{2,0} r^{2}, \quad \chi_{2,0}=B_{2,0} r^{2} .\end{array}\right\}$

Here, $A_{2,0}, B_{2,0}, C_{2,0}, A_{0,0}$, and $D_{0,0}$ are arbitrary constants obeying the relations

$$
\left.\begin{array}{l}
3\left(\sigma^{2} A_{2,0}-2 B_{2,0}\right)-2 K D_{0,0}=0, \\
3\left(B_{2,0}-C_{2,0}\right) \\
\quad+K\left[2 A_{0,0}+\left(2-3 \xi_{S}^{2}\right) A_{2,0}+\Gamma_{1} D_{0,0}\right]=0 .
\end{array}\right\}
$$

Thirdly, from the system of equations associated with $\ell=0$, it follows that

$$
\left.\begin{array}{l}
\xi_{0,0}=A_{0,0} r \\
\Phi_{0,0}^{\prime}=C_{0,0} r^{2}+C_{0,0}^{\prime}, \\
\chi_{0,0}=B_{0,0} r^{2}+B_{0,0}^{\prime} .
\end{array}\right\}
$$

Here, $B_{0,0}, B_{0,0}^{\prime}, C_{0,0}, C_{0,0}^{\prime}$ are arbitrary constants obeying the relations

$$
\left.\begin{array}{l}
3 C_{0,0}+2 \pi G \rho D_{0,0}=0, \\
3 A_{0,0}-D_{0,0}=0, \\
6 B_{0,0}-\left[\sigma^{2}+4 \pi G \rho\right] D_{0,0}=0, \\
B_{0,0}^{\prime}-C_{0,0}^{\prime}+\frac{a^{* 2}}{8} \xi_{\mathrm{S}}^{2}\left(\xi_{\mathrm{S}}^{2}-1\right) \Gamma_{1} K D_{0,0}=0, \\
K A_{2,0}+\left[\frac{\sigma^{2}}{2}+2 \pi G \rho\left(\frac{4}{3}-\Gamma_{1}\right)\right] D_{0,0}=0 .
\end{array}\right\}
$$

Hence, the axisymmetric second-harmonic solutions of the compressible Jeans spheroids take the form

$$
\left.\begin{array}{l}
\delta r\left(r, \theta^{*}, \phi^{*}\right)=A_{2,0} r P_{2}\left(\cos \theta^{*}\right)+A_{0,0} r, \\
\delta \theta^{*}\left(r, \theta^{*}, \phi^{*}\right)=\frac{A_{2,0}}{2} \frac{\mathrm{d} P_{2}\left(\cos \theta^{*}\right)}{\mathrm{d} \theta^{*}}, \\
\delta \phi^{*}\left(r, \theta^{*}, \phi^{*}\right)=0, \\
\alpha\left(r, \theta^{*}, \phi^{*}\right)=D_{0,0}, \\
\Phi^{\prime}\left(r, \theta^{*}, \phi^{*}\right)=C_{2,0} r^{2} P_{2}\left(\cos \theta^{*}\right)+C_{0,0} r^{2}+C_{0,0}^{\prime} \\
P^{\prime}\left(r, \theta^{*}, \phi^{*}\right)=\rho\left[\left(B_{2,0}-C_{2,0}\right) r^{2} P_{2}\left(\cos \theta^{*}\right)\right. \\
\left.\quad+\left(B_{0,0}-C_{0,0}\right) r^{2}+\left(B_{0,0}^{\prime}-C_{0,0}^{\prime}\right)\right] .
\end{array}\right\}
$$

After transforming to prolate spheroidal coordinates and imposing the boundary conditions, one derives the following relations between the constants $C_{2,0}, C_{0,0}, C_{0,0}^{\prime}$, and $D_{0,0}$ :

$$
\left.\begin{array}{l}
C_{0,0}\left(3 \xi_{\mathrm{S}}^{2}-2\right)+C_{2,0}+\frac{12}{a^{2}} C_{0,0}^{\prime}=0 \\
C_{2,0}-\pi G \rho\left(\xi_{\mathrm{S}}^{2}-1\right) \\
\left\{1-\xi_{\mathrm{S}} \ln \frac{\xi_{\mathrm{S}}+1}{\xi_{\mathrm{S}}-1}+\frac{1}{3} \frac{3 \xi_{\mathrm{S}}^{2}-1}{\xi_{\mathrm{S}}^{2}-1}\right. \\
\quad+\frac{3}{4}\left[\frac{\sigma^{2}}{4 \pi G \rho}+\left(\frac{4}{3}-\Gamma_{1}\right)\right]\left(3 \xi_{\mathrm{S}}^{2}-1\right) \xi_{\mathrm{S}} \\
\left.\left[\left(3 \xi_{\mathrm{S}}^{2}-1\right) \ln \frac{\xi_{\mathrm{S}}+1}{\xi_{\mathrm{S}}-1}-6 \xi_{\mathrm{S}}\right]\right\} D_{0,0}=0 .
\end{array}\right\}
$$

Equations (62), (64), and (66) form a homogeneous system of nine equations for the nine unknown constants $A_{2,0}, B_{2,0}, C_{2,0}$, $A_{0,0}, B_{0,0}, C_{0,0}, D_{0,0}, B_{0,0}^{\prime}, C_{0,0}^{\prime}$. The condition for the system to admit of a non-trivial solution yields the quadratic equation for the square of the eigenfrequency

$$
\begin{aligned}
\sigma^{2} & =2 \pi G \rho\left\{\widetilde{Q} \pm\left[\widetilde{Q}^{2}+12 \Gamma_{1} \xi_{\mathrm{S}}\left(\xi_{\mathrm{S}}^{2}-1\right) Q_{2}\left(\xi_{\mathrm{S}}\right)\right.\right. \\
& \left.\left.+8 \frac{\xi_{\mathrm{S}}^{2}\left(\xi_{\mathrm{S}}^{2}-1\right)}{\left(3 \xi_{\mathrm{S}}^{2}-1\right)^{2}}\left[6 \xi_{\mathrm{S}}\left(2-3 \xi_{\mathrm{S}}^{2}\right) Q_{2}\left(\xi_{\mathrm{S}}\right)+\left(4-3 \Gamma_{1}\right)\right]\right]^{1 / 2}\right\},
\end{aligned}
$$

where the function $\widetilde{Q}\left(\xi_{\mathrm{S}}\right)$ is defined as

$$
\widetilde{Q}\left(\xi_{\mathrm{S}}\right)=-3 \xi_{\mathrm{S}}\left(\xi_{\mathrm{S}}^{2}-1\right) Q_{2}\left(\xi_{\mathrm{S}}\right)+\Gamma_{1}-\frac{2 \xi_{\mathrm{S}}^{2}}{3 \xi_{\mathrm{S}}^{2}-1} \text {. }
$$

The values of the squares of the eigenfrequencies resulting from Eq. (67) for $\Gamma_{1}=1.3,4 / 3,1.4,1.5,1.6,5 / 3$ agree with those given by Chandrasekhar \& Lebovitz (1963) in their Table 2 and denoted as $\sigma_{\mathrm{R}}^{2}$ and $\sigma_{\mathrm{S}}^{2}$. The value $\Gamma_{1}=1.6$ is a critical value for the interpretation of the roots of the equation: for $\Gamma_{1}<1.6$, the root associated with the minus sign in the right-hand member of the equation is the root $\sigma_{\mathrm{R}}^{2}$, and the root associated with the plus sign in the right-hand member of the equation is the root $\sigma_{\mathrm{S}}^{2}$, while, for $\Gamma_{1}>1.6$, the reverse holds. 


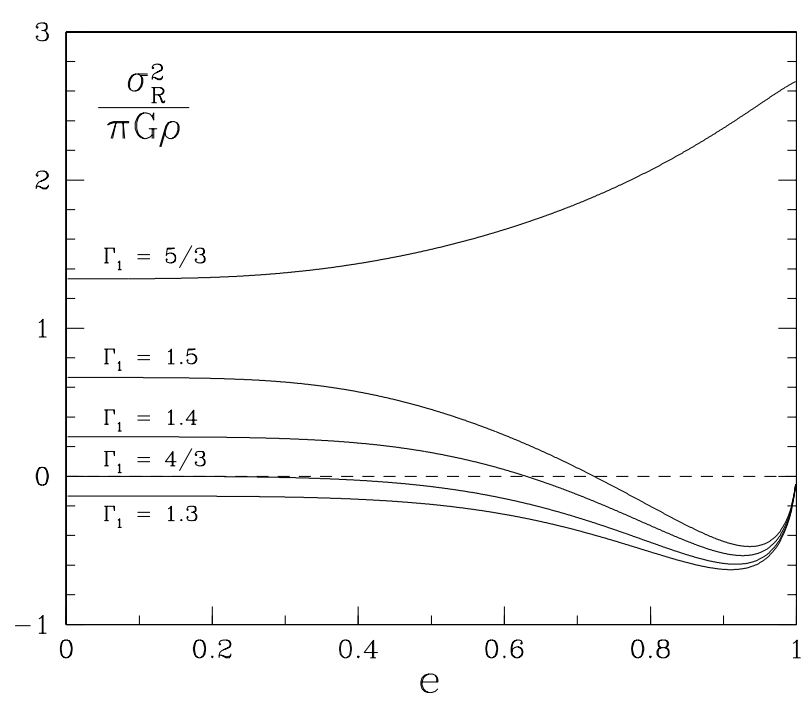

Fig. 4. The eigenvalues $\sigma_{\mathrm{R}}^{2}$ of the second-harmonic $R$-mode in the compressible Jeans spheroid as functions of the eccentricity of the Jeans spheroid, for various values of $\Gamma_{1}$.

For $\Gamma_{1} \neq 1.6$ and Jeans spheroids with a small eccentricity, one derives the following Taylor series for the roots $\sigma_{\mathrm{R}}^{2}$ and $\sigma_{\mathrm{S}}^{2}$ :

$\sigma_{\mathrm{R}}^{2}=4 \pi G \rho\left[\left(\Gamma_{1}-\frac{4}{3}\right)+\frac{4}{81} \frac{14-15 \Gamma_{1}}{8-5 \Gamma_{1}} e^{4}+O\left(e^{6}\right)\right]$,

$\sigma_{\mathrm{S}}^{2}=\frac{16}{15} \pi G \rho\left[1-\frac{13}{21} e^{2}-\frac{1}{189} \frac{86-585 \Gamma_{1}}{8-5 \Gamma_{1}} e^{4}+O\left(e^{6}\right)\right]$.

From the limiting values of the roots $\sigma_{\mathrm{R}}^{2}$ and $\sigma_{\mathrm{S}}^{2}$ for $e \rightarrow 0$, it appears that the root $\sigma_{\mathrm{R}}^{2}$ originates from the square of the eigenfrequency of the fundamental radial mode in the compressible equilibrium sphere with uniform mass density, while the root $\sigma_{\mathrm{S}}^{2}$ originates from the square of the eigenfrequency of the axisymmetric second-degree Kelvin-mode in the same equilibrium sphere.

The variations of $\sigma_{\mathrm{R}}^{2}$ and $\sigma_{\mathrm{S}}^{2}$ as functions of the eccentricity of the Jeans spheroid are represented in Figs. 4 and 5 for various values of $\Gamma_{1}$ different from 1.6.

\section{Test of the validity of the perturbation method}

\subsection{The compressible homogeneous model}

In the case of the compressible homogeneous model that is perturbed by an equilibrium tide, we compare the results determined by means of the first-order perturbation method for the fundamental radial mode and the second-degree Kelvin modes with the analytical solutions established in the previous section for the second-harmonic oscillations of compressible Jeans spheroids with a small eccentricity. For this comparison, we regard the Jeans spheroids as tidally distorted equilibrium configurations that rotate uniformly around an axis perpendicular to the orbital plane and synchronously with the accompanying body, without being perturbed by the axial rotation.

In the homogeneous model, the pressure distribution is given by

$P(r)=\frac{2 \pi G \rho^{2}}{3} R^{2}\left(1-\frac{r^{2}}{R^{2}}\right)$.

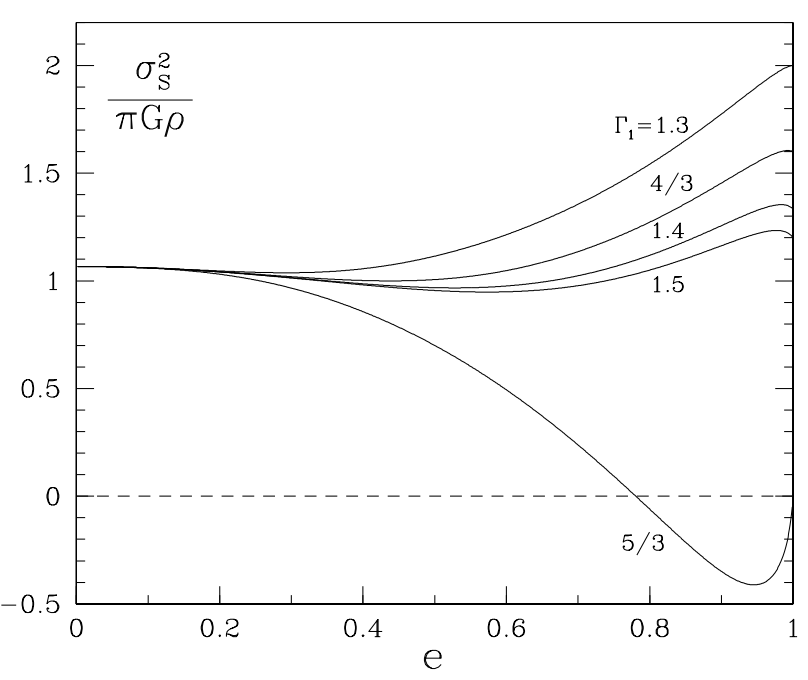

Fig. 5. The eigenvalues $\sigma_{\mathrm{S}}^{2}$ of the second-harmonic $S$-mode in the compressible Jeans spheroid as functions of the eccentricity of the Jeans spheroid, for various values of $\Gamma_{1}$.

With regard to the equilibrium tide, the function $\xi_{\mathrm{st}}(r)$, which appears in Eq. (9) of Paper I for the second-degree components of the tidal displacement, is an exact analytical solution of Clairaut's equation, Eq. (10) of Paper I:

$\xi_{\mathrm{st}}(r)=-\frac{5}{4} r$.

The components of the equilibrium tide with respect to the local coordinate basis $\partial / \partial r, \partial / \partial \theta, \partial / \partial \phi$ are then

$$
\left.\begin{array}{l}
\varepsilon_{\mathrm{T}}(\delta r)_{\mathrm{eq}}(\boldsymbol{r})=-\varepsilon_{\mathrm{T}} \frac{5}{4} r \\
\times\left[Y_{2}(\theta)-\frac{1}{4} Y_{2}^{2}(\theta, \phi)-\frac{1}{4} Y_{2}^{-2}(\theta, \phi)\right], \\
\varepsilon_{\mathrm{T}}(\delta \theta)_{\mathrm{eq}}(\boldsymbol{r})=-\varepsilon_{\mathrm{T}} \frac{5}{8} \\
\quad \times\left[\frac{\partial Y_{2}(\theta)}{\partial \theta}-\frac{1}{4} \frac{\partial Y_{2}^{2}(\theta, \phi)}{\partial \theta}-\frac{1}{4} \frac{\partial Y_{2}^{-2}(\theta, \phi)}{\partial \theta}\right], \\
\varepsilon_{\mathrm{T}}(\delta \phi)_{\mathrm{eq}}(\boldsymbol{r})=-\varepsilon_{\mathrm{T}} \frac{5}{8} \\
\quad \times \frac{1}{\sin ^{2} \theta}\left[\frac{\partial Y_{2}(\theta)}{\partial \phi}-\frac{1}{4} \frac{\partial Y_{2}^{2}(\theta, \phi)}{\partial \phi}-\frac{1}{4} \frac{\partial Y_{2}^{-2}(\theta, \phi)}{\partial \phi}\right] .
\end{array}\right\}
$$

In view of the comparison of the results of the first-order perturbation method with those established for the second-harmonic oscillations of compressible Jeans spheroids with a small eccentricity, we observe that the expansion parameter $\varepsilon_{\mathrm{T}}$ adopted in the perturbation method is related to the eccentricity $e$ of the Jeans spheroid through Eqs. (12) and (13). For small values of the eccentricity, one has

$\varepsilon_{\mathrm{T}}=\frac{2}{15} e^{2}\left[1+O\left(e^{2}\right)\right]$.

Hence, the application of the first-order perturbation method to the oscillations of the compressible homogeneous model is equivalent to the approximation of the oscillations of the compressible Jeans spheroids that is exact to the first order in the square of the eccentricity. 


\subsubsection{The perturbed fundamental radial mode}

From the perturbation method developed in Paper I, it follows that, for any radial mode $n$ of an arbitrary stellar model, the first-order perturbation of the eigenfrequency due to an equilibrium tide is identically zero. The expansion for the square of the eigenfrequency of a radial mode is thus of the form

$\sigma_{r}^{2}=\sigma_{r, 0}^{2}\left[1+O\left(\varepsilon_{\mathrm{T}}^{2}\right)\right]$,

where $\sigma_{r, 0}^{2}$ is the square of the eigenfrequency of the radial mode in the unperturbed star. The expansion can equivalently be written as

$\sigma_{r}^{2}=\sigma_{r, 0}^{2}\left[1+O\left(e^{4}\right)\right]$.

In the particular case of the fundamental radial mode of the compressible homogeneous model, the square of the eigenfrequency is given by

$\sigma_{r, 0}^{2}=4 \pi G \rho\left(\Gamma_{1}-\frac{4}{3}\right)$.

Hence, for the perturbed fundamental radial mode of the compressible homogeneous equilibrium sphere, Expansion (76) agrees, up to order $e^{2}$, with Expansion (69) for the square of the eigenfrequency of the $R$-mode of the compressible Jeans spheroids.

\subsubsection{The perturbed second-degree Kelvin modes}

Here the subscript $n$ introduced in Paper I denotes the seconddegree Kelvin modes of the compressible homogeneous model. For these modes, the functions $\xi_{n, 2}(r)$ and $\eta_{n, 2}(r)$, which appear in Eqs. (71) of Paper I for the components of the Lagrangian displacement at order zero, are simply given by

$\xi_{n, 2}(r)=r, \quad \eta_{n, 2}(r)=\frac{r^{2}}{2}$.

Using the foregoing functions, we derived by means of Eq. (78) of Paper I that, for $\ell=2$,

$H_{n, 0 ; n, 0}=\frac{65}{28}$.

According to Eq. (116) and Fig. 2 of Paper I, one finds the following first-order corrections to the eigenfrequency for $\widetilde{m}=$ $0,|\widetilde{m}|=1,|\widetilde{m}|=2$, respectively:

$\frac{\sigma_{n, 1}^{(1)}}{\sigma_{n, 0}}=-\frac{65}{28}, \quad \frac{\sigma_{n, 1}^{(2)}}{\sigma_{n, 0}}=-\frac{65}{56}, \quad \frac{\sigma_{n, 1}^{(3)}}{\sigma_{n, 0}}=\frac{65}{28}$.

By using

$\sigma_{n, 0}^{2}=\frac{16 \pi G \rho}{15}$,

and passing on from the expansion parameter $\varepsilon_{\mathrm{T}}$ to the eccentricity $e$ of the Jeans spheroid, one derives the following expansions for the squares of the eigenfrequencies:

$\sigma_{n}^{2}=\frac{16 \pi G \rho}{15}\left[1-\frac{13}{21} e^{2}+O\left(e^{4}\right)\right]$, for $\widetilde{m}=0$,

$\sigma_{n}^{2}=\frac{16 \pi G \rho}{15}\left[1-\frac{13}{42} e^{2}+O\left(e^{4}\right)\right]$, for $|\widetilde{m}|=1$,

$\sigma_{n}^{2}=\frac{16 \pi G \rho}{15}\left[1+\frac{13}{21} e^{2}+O\left(e^{4}\right)\right]$, for $|\widetilde{m}|=2$.
After comparison, one reaches the conclusion that the foregoing expansions for $\sigma_{n}^{2}$ agree, up to order $e^{2}$, with the expansions given by Eqs. (70), (44), and (47) for the squares of the eigenfrequencies of the second-harmonic oscillations of the compressible Jeans spheroids.

We also verify that the zero-order components of the Lagrangian displacement determined for the second-degree Kelvin modes of the compressible homogeneous model are equivalent to the components of the Lagrangian displacement derived, at the same degree of approximation, for the secondharmonic oscillations of the compressible Jeans spheroids.

In the perturbation method developed in Paper I, we initially determined the zero-order components of the Lagrangian displacement with respect to a system of spherical coordinates whose polar axis coincides with the star's rotation axis perpendicular to the orbital plane. Subsequently, we moved on to the zero-order components with respect to a system of spherical coordinates whose polar axis coincides with the axis pointing from the star's mass centre towards the companion. In the latter case, the zero-order components of the Lagrangian displacement in a second-degree oscillation mode are given by Eqs. (123) of Paper I. We here apply these equations to the second-degree Kelvin modes of the compressible homogeneous model that is perturbed by an equilibrium tide.

\section{The second-degree Kelvin mode associated with $\widetilde{m}=0$}

For the second-degree Kelvin mode associated with $\widetilde{m}=0$, the zero-order components of the Lagrangian displacement are

$(\delta r)_{n}(\boldsymbol{r})=-2 A_{2} r P_{2}\left(\cos \theta^{*}\right)$,

$\left(\delta \theta^{*}\right)_{n}(\boldsymbol{r})=-A_{2} \frac{\mathrm{d} P_{2}\left(\cos \theta^{*}\right)}{\mathrm{d} \theta^{*}}$,

$\left(\delta \phi^{*}\right)_{n}(\boldsymbol{r})=0$

where $A_{2}$ is an arbitrary constant.

On the other hand, the components of the Lagrangian displacement in the second-harmonic oscillations of the compressible Jeans spheroids associated with $\widetilde{m}=0$ are given by the first three Eqs. (65). From the relations given by Eqs. (64) and the Taylor series for $\sigma_{\mathrm{S}}^{2}$ near $e=0$, one can derive the relation between $A_{2,0}$ and $A_{0,0}$. At the lowest order of approximation, this is

$A_{2,0}=-\frac{9}{10}\left(8-5 \Gamma_{1}\right) \frac{1}{e^{2}} A_{0,0}$.

Hence, the term with the constant $A_{0,0}$ in the solution for $\delta r$ is of a higher order in $e^{2}$ than the term with the constant $A_{2,0}$ and, consequently, it can be discarded. The components $\delta r, \delta \theta^{*}, \delta \phi^{*}$ of the Lagrangian displacement in the axisymmetric secondharmonic oscillation of a compressible Jeans spheroid with a small eccentricity then take the form

$$
\begin{aligned}
& \delta r=A_{2,0} r P_{2}\left(\cos \theta^{*}\right), \\
& \delta \theta^{*}=\frac{A_{2,0}}{2} \frac{\mathrm{d} P_{2}\left(\cos \theta^{*}\right)}{\mathrm{d} \theta^{*}}, \\
& \delta \phi^{*}=0 .
\end{aligned}
$$


From the comparison, it follows that the zero-order solution for the axisymmetric second-degree Kelvin mode in the compressible homogeneous model that is distorted by an equilibrium tide is equivalent to the lowest-order solution for the axisymmetric second-harmonic oscillation in a compressible Jeans spheroid with a small eccentricity.

\section{The second-degree Kelvin modes associated with $\widetilde{m}= \pm 1$}

For the second-degree Kelvin modes associated with $\widetilde{m}= \pm 1$, the zero-order components of the Lagrangian displacement are

$$
\begin{aligned}
& (\delta r)_{n}(\boldsymbol{r})=r P_{2}^{1}\left(\cos \theta^{*}\right)\left[B_{2}^{*} \exp \left(\mathrm{i} \phi^{*}\right)\right. \\
& \left.+C_{2}^{*} \exp \left(-\mathrm{i} \phi^{*}\right)\right], \\
& \left(\delta \theta^{*}\right)_{n}(\boldsymbol{r})=\frac{1}{2} \frac{\mathrm{d} P_{2}^{1}\left(\cos \theta^{*}\right)}{\mathrm{d} \theta^{*}}\left[B_{2}^{*} \exp \left(\mathrm{i} \phi^{*}\right)\right. \\
& \left.+C_{2}^{*} \exp \left(-\mathrm{i} \phi^{*}\right)\right], \\
& \left(\delta \phi^{*}\right)_{n}(\boldsymbol{r})=\frac{\mathrm{i}}{2} \frac{P_{2}^{1}\left(\cos \theta^{*}\right)}{\sin ^{2} \theta^{*}}\left[B_{2}^{*} \exp \left(\mathrm{i} \phi^{*}\right)\right. \\
& \left.-C_{2}^{*} \exp \left(-\mathrm{i} \phi^{*}\right)\right],
\end{aligned}
$$

where $B_{2}^{*}$ and $C_{2}^{*}$ are arbitrary constants.

These solutions are equivalent to those given by Eqs. (42) for the components of the Lagrangian displacement in the second-harmonic oscillations associated with $\widetilde{m}= \pm 1$ of a Jeans spheroid with a small eccentricity.

\section{The second-degree Kelvin modes associated with $\widetilde{\mathrm{m}}= \pm 2$}

For the second-degree Kelvin modes associated with $\widetilde{m}= \pm 2$, the zero-order components of the Lagrangian displacement are

$$
\begin{aligned}
& (\delta r)_{n}(\boldsymbol{r})=r P_{2}^{2}\left(\cos \theta^{*}\right)\left[D_{2}^{*} \exp \left(2 \mathrm{i} \phi^{*}\right)\right. \\
& \left.+E_{2}^{*} \exp \left(-2 \mathrm{i} \phi^{*}\right)\right], \\
& \left(\delta \theta^{*}\right)_{n}(\boldsymbol{r})=\frac{1}{2} \frac{\mathrm{d} P_{2}^{2}\left(\cos \theta^{*}\right)}{\mathrm{d} \theta^{*}}\left[D_{2}^{*} \exp \left(2 \mathrm{i} \phi^{*}\right)\right. \\
& \left.+E_{2}^{*} \exp \left(-2 \mathrm{i} \phi^{*}\right)\right], \\
& \left(\delta \phi^{*}\right)_{n}(\boldsymbol{r})=\mathrm{i} \frac{P_{2}^{2}\left(\cos \theta^{*}\right)}{\sin ^{2} \theta^{*}}\left[D_{2}^{*} \exp \left(2 \mathrm{i} \phi^{*}\right)\right. \\
& \left.-E_{2}^{*} \exp \left(-2 \mathrm{i} \phi^{*}\right)\right],
\end{aligned}
$$

where $D_{2}^{*}$ and $E_{2}^{*}$ are arbitrary constants.

\begin{tabular}{|c|c|c|c|c|}
\hline$\ell$ & mode & $\omega_{n, 0}^{2}$ & $\frac{\sigma_{n, 1}^{(1)}}{\sigma_{n, 0}}$ & $\left(\frac{\sigma_{n, 1}^{(1)}}{\sigma_{n, 0}}\right) /\left(\frac{\sigma_{n, 1}^{(1)}}{\sigma_{n, 0}}\right)_{\mathrm{S}}$ \\
\hline \multirow{9}{*}{1} & $p_{6}$ & 94.252 & -0.8476 & -2.018 \\
\hline & $p_{5}$ & 71.289 & -0.8456 & -2.011 \\
\hline & $p_{4}$ & 51.505 & -0.8413 & -2.005 \\
\hline & $p_{3}$ & 34.919 & -0.8322 & -2.001 \\
\hline & $p_{2}$ & 21.552 & -0.8121 & -1.998 \\
\hline & $p_{1}$ & 11.404 & -0.7629 & -1.997 \\
\hline & $g_{1}$ & 2.5159 & -0.1260 & -1.871 \\
\hline & $g_{2}$ & 1.2857 & -0.1377 & -1.966 \\
\hline & $g_{3}$ & 0.77573 & -0.1451 & -1.972 \\
\hline \multirow{10}{*}{2} & $p_{6}$ & 104.88 & -0.6047 & -2.017 \\
\hline & $p_{5}$ & 80.567 & -0.6033 & -2.011 \\
\hline & $p_{4}$ & 59.428 & -0.6007 & -2.005 \\
\hline & $p_{3}$ & 41.473 & -0.5954 & -1.999 \\
\hline & $p_{2}$ & 26.723 & -0.5836 & -1.992 \\
\hline & $p_{1}$ & 15.264 & -0.5444 & -1.979 \\
\hline & $f$ & 8.1753 & -0.3089 & -1.911 \\
\hline & $g_{1}$ & 4.9146 & -0.1014 & -1.931 \\
\hline & $g_{2}$ & 2.8282 & -0.07021 & -1.976 \\
\hline & $g_{3}$ & 1.8223 & -0.06529 & -1.981 \\
\hline \multirow{10}{*}{3} & $p_{6}$ & 114.60 & -0.5657 & -2.016 \\
\hline & $p_{5}$ & 88.999 & -0.5648 & -2.011 \\
\hline & $p_{4}$ & 66.566 & -0.5629 & -2.005 \\
\hline & $p_{3}$ & 47.316 & -0.5593 & -2.001 \\
\hline & $p_{2}$ & 31.260 & -0.5515 & -1.995 \\
\hline & $p_{1}$ & 18.444 & -0.5281 & -1.989 \\
\hline & $f$ & 9.4138 & -0.3809 & -1.990 \\
\hline & $g_{1}$ & 6.7670 & -0.08935 & -1.967 \\
\hline & $g_{2}$ & 4.2364 & -0.05583 & -1.965 \\
\hline & $g_{3}$ & 2.8867 & -0.05197 & -1.962 \\
\hline
\end{tabular}

The solutions are now equivalent to those given by Eqs. (45) for the components of the Lagrangian displacement in the second-harmonic oscillations associated with $\widetilde{m}= \pm 2$ of a Jeans spheroid with a small eccentricity.

\subsection{The polytropic model with index $n=3$}

In the case of the polytropic model with index $n=3$, we compare the tidal corrections to the eigenfrequencies that are determined by means of the perturbation method developed in
Table 1. The relative first-order correction $\sigma_{n, 1}^{(1)} / \sigma_{n, 0}$ for various modes of the polytropic model with index $n=3$ and the ratio of this relative correction to its value in Saio's paper.

Paper I, with results that were obtained by Saio (1981) for several lower-order modes $n$ belonging to the degrees $\ell=1,2,3$. In his investigation, Saio used a perturbation method developed earlier by Smeyers \& Denis (1971) and Denis (1972), whose applicability is restricted to polytropic models.

In Table 1, we present the dimensionless squares of the eigenfrequencies $\omega_{n, 0}^{2}=\left(R_{1}^{3} /\left(G M_{1}\right)\right) \sigma_{n, 0}^{2}$ and the ratios of the single first-order correction $\sigma_{n, 1}^{(1)}$ to the zero-order eigenfrequency $\sigma_{n, 0}$. One observes that the ratios $\sigma_{n, 1}^{(1)} / \sigma_{n, 0}$ are all negative.

According to Saio's paper, the eigenfrequency of an oscillation mode that is perturbed by an equilibrium tide can be expressed as follows up to the second order in the star's angular velocity of rotation $\Omega$ :

$\sigma=\sigma_{0}\left[1+\frac{3}{2} \frac{M_{2}}{M_{1}+M_{2}}\left(X_{2}+m^{2} Y_{2}\right)\left(\frac{\Omega}{\sigma_{0}}\right)^{2}\right]$. 
Here $m$ is the azimuthal number of the spherical harmonic of the angular variables $\theta$ and $\phi$, whose polar axis coincides with the rotational axis perpendicular to the orbital plane. Furthermore, $X_{2}$ and $Y_{2}$ are numerical quantities which depend on the mode considered. Since, for an equilibrium tide, the star's angular velocity of rotation is related to the parameter $\varepsilon_{\mathrm{T}}$ through Kepler's third law as

$\Omega^{2}=\frac{G M_{1}}{R_{1}^{3}} \frac{M_{1}+M_{2}}{M_{2}} \varepsilon_{\mathrm{T}}$,

Eq. (88) can be rewritten as

$\sigma=\sigma_{0}\left[1+\varepsilon_{\mathrm{T}} \frac{3}{2 \sigma_{0}^{2}}\left(X_{2}+m^{2} Y_{2}\right) \frac{G M_{1}}{R_{1}^{3}}\right]$.

From the numerical results tabulated in Saio's paper, it follows that, for the modes of a given degree $\ell$, the quantity $Y_{2}$ is proportional to the quantity $X_{2}$. The constant relating the two quantities is $-3 / 2,-1 / 2$, and $-1 / 4$, respectively.

By using these relations, we have derived the following equations for the relative first-order corrections to the eigenfrequency: for modes belonging to $\ell=1$,

$\left(\frac{\sigma_{n, 1}^{(1)}}{\sigma_{n, 0}}\right)_{\mathrm{S}}=\frac{3}{2 \omega_{0}^{2}} X_{2}, \quad\left(\frac{\sigma_{n, 1}^{(2)}}{\sigma_{n, 0}}\right)_{\mathrm{S}}=-\frac{3}{4 \omega_{0}^{2}} X_{2} ;$

for modes belonging to $\ell=2$,

$\left.\begin{array}{l}\left(\frac{\sigma_{n, 1}^{(1)}}{\sigma_{n, 0}}\right)_{\mathrm{S}}=\frac{3}{2 \omega_{0}^{2}} X_{2}, \quad\left(\frac{\sigma_{n, 1}^{(2)}}{\sigma_{n, 0}}\right)_{\mathrm{S}}=-\frac{3}{4 \omega_{0}^{2}} X_{2}, \\ \left(\frac{\sigma_{n, 1}^{(3)}}{\sigma_{n, 0}}\right)_{\mathrm{S}}=-\frac{3}{2 \omega_{0}^{2}} X_{2} ;\end{array}\right\}$

and, for modes belonging to $\ell=3$,

$$
\left.\begin{array}{l}
\left(\frac{\sigma_{n, 1}^{(1)}}{\sigma_{n, 0}}\right)_{\mathrm{S}}=\frac{3}{2 \omega_{0}^{2}} X_{2}, \quad\left(\frac{\sigma_{n, 1}^{(2)}}{\sigma_{n, 0}}\right)_{\mathrm{S}}=\frac{9}{8 \omega_{0}^{2}} X_{2}, \\
\left(\frac{\sigma_{n, 1}^{(3)}}{\sigma_{n, 0}}\right)_{\mathrm{S}}=0, \quad\left(\frac{\sigma_{n, 1}^{(4)}}{\sigma_{n, 0}}\right)_{\mathrm{S}}=-\frac{15}{8 \omega_{0}^{2}} X_{2} .
\end{array}\right\}
$$

The superscript on $\sigma_{n, 1}$ refers to the value of $|\widetilde{m}|+1$, as before.

The foregoing equations lead to relations between the firstorder corrections to the eigenfrequency of a mode $n$ that fully agree with the relations we derived in Paper I by means of the perturbation method.

Next, we have used the values of $X_{2}$ tabulated in Saio's paper in order to determine the values of the relative firstorder corrections $\left(\sigma_{n, 1}^{(1)} / \sigma_{n, 0}\right)_{\mathrm{S}}$ for the low-degree and low-order modes considered by that author. The ratios of the relative first-order correction $\left(\sigma_{n, 1}^{(1)} / \sigma_{n, 0}\right)$ determined by means of our perturbation method to the corresponding first-order correction $\left(\sigma_{n, 1}^{(1)} / \sigma_{n, 0}\right)_{\mathrm{S}}$ are listed in the last column of Table 1. From these ratios, it appears that our first-order corrections are nearly equal to minus two times the first-order corrections based on Saio's results:

$$
\frac{\sigma_{n, 1}^{(1)}}{\sigma_{n, 0}} \simeq-2\left(\frac{\sigma_{n, 1}^{(1)}}{\sigma_{n, 0}}\right)_{\mathrm{S}} \text {. }
$$

Similar relations are found for the first-order corrections $\sigma_{n, 1}^{(2)}$, $\sigma_{n, 1}^{(3)}$, and $\sigma_{n, 1}^{(4)}$.

The discrepancy seems to be related to the determination of our quantity $H_{n, 0 ; n, 0}$ or/and that of Saio's quantity $X_{2}$. Despite repeated verifications of our derivations of the quantities $H_{n, 0 ; n, 0}$ and of our numerical code, we have not been able to remove the discrepancy.

We may observe that, in the case of the compressible homogeneous model, the quantities $H_{n, 0 ; n, 0}$ and our numerical code did lead to first-order corrections to the eigenfrequency that all agreed with the corrections derived for the second-harmonic oscillations of the compressible Jeans spheroids at the same degree of approximation, as shown above. We also observe that the quantities $H_{n, 0 ; n, 0}$ determined for the polytropic model with index $n=3$ are positive as are those determined for the compressible homogeneous model. However, according to the results given by Saio, the quantities $H_{n, 0 ; n, 0}$ determined for the polytropic model with index $n=3$ should be negative.

\section{Concluding remarks}

In this investigation, we have tested the validity of the firstorder perturbation method developed in Paper I for the determination of the effects of an equilibrium tide on linear, isentropic oscillations of a component of a close binary. We have performed the test for two simple models for which results on tidal perturbations of linear, isentropic oscillations are available by other means: the compressible homogeneous model and the polytropic model with index $n=3$.

First, in the case of the compressible homogeneous model, we have compared the results that are obtained by the application of the perturbation method to the fundamental radial mode and the second-degree Kelvin modes, with the secondharmonic oscillations of compressible Jeans spheroids with a small eccentricity. To this end, we have previously derived exact analytical solutions for the squares of the eigenfrequencies and for the eigenfunctions of the second-harmonic oscillations of the compressible Jeans spheroids, for any value of the eccentricity. From our severe test, we have concluded that the perturbed eigenfrequencies of the fundamental radial mode and the second-degree Kelvin modes of the compressible homogeneous model fully agree with the eigenfrequencies of the second-harmonic oscillations of the compressible Jeans spheroids, up to the second order in the small eccentricity of the spheroids. For the same modes, we have also concluded that the lowest-order Lagrangian displacements in the tidally perturbed homogeneous model are equivalent to those in the Jeans spheroids.

Secondly, in the case of the polytropic model with index $n=3$, we have compared the results that are obtained by the application of our perturbation method to various low-order and low-degree modes with results given by Saio (1981) for the same modes in a rotationally and tidally polytropic model. Saio's results were obtained by the use of an earlier perturbation method due to Smeyers \& Denis (1971) and Denis (1972). By an analysis of Saio's results, we have recovered the relations between the first-order tidal corrections to the eigenfrequencies established in Paper I for modes of any stellar model 
that belong to one of the low degrees $\ell=1,2,3$. However, from the comparison of the values of the first-order corrections $\sigma_{n, 1}$ to the eigenfrequency, it appears that the first-order corrections determined by means of our perturbation method differ by nearly a factor -2 from those of Saio. We have been unable to identify the source of the discrepancy. Nevertheless, we believe our results to be correct if only because they are consistent with the eigenfrequencies and eigenfunctions for the compressible Jeans spheroids with a small eccentricity.

Acknowledgements. The authors acknowledge the stimulating remarks of the referee Dr. M. Clement, which allowed them to improve the presentation of the paper.

\section{References}

Chandrasekhar, S. 1969, Ellipsoidal Figures of Equilibrium, The Silliman Foudation Lectures (New Haven: Yale University Press) Chandrasekhar, S., \& Lebovitz, N. R. 1963, ApJ, 137, 1172

Denis, J. 1972, A\&A, 20, 151

Morse, P. M., \& Feshbach, H. 1953, Methods of Theoretical Physics, International Series in Pure and Applied Physics (New York: McGraw-Hill)

Reyniers, K., \& Smeyers, P. 2003, A\&A, 404, 1051

Saio, H. 1981, ApJ, 244, 299

Smeyers, P. 1986, A\&A, 160, 385

Smeyers, P., \& Denis, J. 1971, A\&A, 14, 311 